\title{
Combination Prediction Model of Flight Mishap Based on Results of Single Model
}

\author{
XuSheng Gan1,a, Hong Qu2,b, Jing-Shun Duanmu 3,c \\ 1 XiJing College, Xi'an Shaanxi, 710123, China \\ 2 Air Traffic Control and Navigation College, \\ Air Force Engineering University, \\ Xi'an, Shaanxi, 710051, China \\ 3 Material Management and Safety Engineering College, \\ Air Force Engineering \\ University, Xi'an, Shaanxi, 710051, China \\ a ganxusheng123@163.com, bqh1122@163.com, cdmjs3836@163.com
}

\begin{abstract}
To use the single prediction method to forecast the flight mishap, due to the limitation of single prediction method, there exist many advantages such as instable calculation and uncertain result. The combination model based on the prediction results of the single model is introduced in flight mishap prediction. An application example shows that the combination model based on the introduced method has a good prediction accuracy to flight mishap with high stability.

Keywords: Combination Prediction; Flight Mishap; Weighted Coefficient; Arithmetic Average Method; ARIMA

\section{Introduction}

The prediction of flight mishap is to carry through reasonable judgment to flight mishap that has not currently occurred yet or is not clear in advance based on the historical safety data. It is an important means of the safety management as well as decision-making. The occurrence of flight mishap is affected and restricted by some influence factors of the system that shows the intricate relationships each other with linear characteristic and nonlinear law, this is a fatal challenge to accurately predict the flight mishap, and also is a problem to be solved [1][2].

In 1969, Bates and Granger first proposed a prediction method that combines the several prediction methods [3]. Each prediction method has the unique information characteristics and different assumptions, depict the internal structure of prediction objects from different angles. If the models or methods with the larger error are simply discarded, some useful predictive information may lose. The combination prediction based on the results is to seek the combination and application of some suitable prediction methods according to the requirement, in order to improve the prediction accuracy and reliability.
\end{abstract}




\section{Arithmetic average method}

The arithmetic average method, also known as the equal weighted average method, is a mostly used combination prediction method. In the method, each weighted coefficient is directly equal to

$$
w_{i}=\frac{1}{m}, i=1,2, \cdots, m
$$

(1)

$$
\begin{aligned}
& \text { Obviously } \\
& \qquad \sum_{i=1}^{m} w_{i}=1, w_{i} \geq 0, i=1,2, \cdots, m
\end{aligned}
$$

The weighted coefficients of m single prediction method included in arithmetic average method are completely equal, namely each single prediction method is all treated in the same way. In general, we use it under the condition of close error variance between these single model and unclear single model accuracy.

Due to simple calculation and nonnegative weighting coefficients, the arithmetic average method is widely used in prediction field. The actual research shows that, using a method to forecast, has some risk, and the average prediction several methods can reduce this kind of risk, rather than just a single prediction method. Though the arithmetic average method is the most simple combination prediction method, but it usually is more prudent than a single prediction method. But when the accuracy of prediction model is known, to carry out combination prediction, a natural idea is to give the larger weighted coefficient to the single prediction model with the high precision.

\section{Error square sum reciprocal method}

The prediction error square sum method, also known as the variance reciprocal method, is the improvement of equivalence weighted average method. The prediction error square sum is a an indicator that reflects the prediction precision, the accuracy of each single prediction model is different, the larger the prediction error square sum, lower the accuracy of the prediction model, the weighted coefficient should be given more small; otherwise, the weighted coefficient is larger.

$$
w_{i}=\frac{E_{i i}^{-1}}{\sum_{i=1}^{m} E_{i i}^{-1}}, i=1,2, \cdots, m
$$

(3)

\section{Obviously}




$$
\sum_{i=1}^{m} w_{i}=1, w_{i} \geq 0, i=1,2, \cdots, m
$$

(4)

$E_{i i}$ is the prediction error square sum of i-th single prediction model:

$$
E_{i i}=\sum_{i=1}^{N}\left(e_{t}^{(i)}\right)^{2}=\sum_{i=1}^{N}\left(x_{t}-\hat{x}_{t}^{(i)}\right)^{2}
$$

where $\hat{x}_{t}^{(i)}$ is the prediction value of i-th single prediction method at time t. ${ }_{t}$ is the observation value of prediction index at time $t, e_{t}^{(i)}=x_{t}-\hat{x}_{t}^{(i)}$ is the prediction error of i-th single method at time $t$.

In the above error square sum reciprocal method, the weighted coefficients are assigned by the error square sum of single prediction model, Similarly, error standard deviation can also be used to assign the weighted coefficients. The calculation formula of the error standard deviation reciprocal method is follows as

$$
w_{i}=\frac{E_{i i}^{-1 / 2}}{\sum_{i=1}^{m} E_{i i}^{-1 / 2}}, i=1,2, \cdots, m
$$

(6)

\section{Obviously}

$$
\sum_{i=1}^{m} w_{i}=1, w_{i} \geq 0, i=1,2, \cdots, m
$$

\section{Practical example}

For the historical flight mishap data of USAF from 1980 to 1996 as shown in Table 1, Gray method [4], simple regression method and ARIMA method [5] and their different combination form is respectively used to establish the prediction model of flight mishap, and then adopt the building model to predict flight mishap of 1997 and 1998 years for model validation.

First, calculate the weighted coefficient according to aforementioned allocation method, for arithmetic average method, the combination coefficient can simply be set as

$$
w_{1}=1 / 3, w_{2}=1 / 3, w_{3}=1 / 3
$$

For error square sum reciprocal method, by the following formula

$$
E_{i i}=\sum_{t=1}^{12}\left(e_{t}^{(i)}\right)^{2}=\sum_{t=1}^{12}\left(x_{t}-\hat{x}_{t}^{(i)}\right)^{2}, i=1,2,3
$$


We can calculate $E_{11}=567.9907, E_{22}=566.9626, E_{33}=326.2261$, then the obtained combination coefficient is respectively

$$
w_{1}=0.2672, w_{2}=0.2677, w_{3}=0.4652
$$

Similarly, use the error standard deviation reciprocal method to calculate the combination coefficient

$$
w_{1}=0.3012, w_{2}=0.3014, w_{3}=0.3974
$$

Table 1 Comparison of fitting result between 3 single prediction model

\begin{tabular}{ccccc}
\hline Years & Actual value & Grey model & Simple regression model & ARIMA(4,1,0) model \\
\hline 1980 & 137 & 137.0000 & 114.0230 & Null \\
1981 & 134 & 106.6592 & 107.0933 & Null \\
1982 & 94 & 100.0879 & 100.5847 & Null \\
1983 & 76 & 93.9215 & 94.4717 & Null \\
1984 & 84 & 88.1351 & 88.7302 & Null \\
1985 & 78 & 82.7051 & 83.3376 & 82.5766 \\
1986 & 78 & 77.6096 & 78.2728 & 77.0913 \\
1987 & 56 & 72.8281 & 73.5158 & 68.9936 \\
1988 & 79 & 68.3412 & 69.0479 & 88.2680 \\
1989 & 60 & 60.1796 & 64.8515 & 63.2166 \\
1990 & 64 & 64.1307 & 60.9102 & 66.9445 \\
1991 & 57 & 56.4720 & 57.2084 & 59.0891 \\
1992 & 59 & 52.9927 & 53.7315 & 59.9775 \\
1993 & 49 & 49.7279 & 50.4660 & 50.6766 \\
1994 & 51 & 46.6642 & 47.3989 & 53.6792 \\
1995 & 51 & 43.7892 & 44.5183 & 47.0757 \\
1996 & 38 & 41.0913 & 41.8127 & 38.0000 \\
\hline
\end{tabular}

So through the arithmetic average method, error square sum reciprocal method and error standard deviation reciprocal method the obtained weighted coefficients are shown Table 2.

Table 2 Allocation table on weighted coefficients 
Weight coefficient allocation Grey model Simple regression model ARIMA $(4,1,0)$ model

\begin{tabular}{cccc}
\hline Arithmetic average method & $1 / 3$ & $1 / 3$ & $1 / 3$ \\
Error square sum reciprocal method & 0.2672 & 0.2677 & 0.4652 \\
Error standard deviation reciprocal method & 0.3012 & 0.3014 & 0.3974 \\
\hline
\end{tabular}

Then Gray GM $(1,1)$ model, simple regression model and ARIMA model is built respectively according to the raw historical data as shown in Table 1 . The established model is as follows:

GM $(1,1)$ model

$$
\begin{aligned}
& x(t+1)=1868.2-1731.2 e^{-0.0636 t}, \quad t=0,1, \cdots, 16 \\
& \text { Simple regression model } \\
& x(t)=121.4011 e^{-0.0627 t}, \quad t=1,2, \cdots, 17 \\
& \text { ARIMA }(4,1,0) \text { model } \\
& \Delta x_{t}=-3.2142-1.2540 \Delta x_{t-1}-0.6240 \Delta x_{t-2}-0.1799 \Delta x_{t-3}+0.0388 \Delta x_{t-4}
\end{aligned}
$$

The above model is used to fit the original flight mishap data, the obtained fitting values are shown in Table 1. From the index value of model evaluation given in Table 3, it can be seen that, for the prediction problem of flight mishap, the accuracy of combination prediction method is better than single prediction method as a whole, and ARIMA method is more accurate than the other two single methods. In the combination prediction method, the method determining weight coefficient by the error information has higher accuracy than the simple arithmetic average method, especially the error square sum reciprocal method has the highest precision. The comparison of prediction results and the relative error from 1997 to 1998 is shown In Table 4. ARIMA method has its unique advantage on generalization ability. However, the combination prediction method has a strong stability and anti - interference. Need to add is, any prediction methods are not universal, has advantages, also has its limitations. Under the undetermined model in advance, singly using a method to predict, is bound to assume certain risks, and the combination prediction method can be greatly reduced or avoid these risks, is also the most insurance, the most secure choice.

Table 3 Comparison of fitting error between single and combination method 


\begin{tabular}{cccc}
\hline Prediction method & MAE & MAPE & MSE \\
\hline Gray method & 5.2029 & 0.0886 & 47.3326 \\
Simple regression method & 5.1548 & 0.0884 & 47.2469 \\
ARIMA method & 3.7712 & 0.0614 & 27.1855 \\
Arithmetic average method & 3.7835 & 0.0658 & 30.1726 \\
Error square sum reciprocal method & 3.2581 & 0.0573 & 26.3269 \\
Error standard deviation reciprocal method & 3.5282 & 0.0617 & 28.0909 \\
\hline
\end{tabular}

Table 4 Comparison of prediction result between single and combination method

\begin{tabular}{|c|c|c|c|c|c|c|c|}
\hline Years & $\begin{array}{l}\text { Actual } \\
\text { value }\end{array}$ & $\begin{array}{l}\text { Grey } \\
\text { method }\end{array}$ & $\begin{array}{l}\text { Simple } \\
\text { regression } \\
\text { method }\end{array}$ & $\begin{array}{l}\text { ARIMA } \\
(4,1,0) \\
\text { method }\end{array}$ & $\begin{array}{l}\text { Arithmetic } \\
\text { average } \\
\text { method }\end{array}$ & $\begin{array}{c}\text { Error square } \\
\text { sum reciprocal } \\
\text { method }\end{array}$ & $\begin{array}{c}\text { Error standard } \\
\text { deviation reciprocal } \\
\text { method }\end{array}$ \\
\hline 1997 & 44 & $\begin{array}{l}38.5597 \\
12.36 \%\end{array}$ & $\begin{array}{l}39.2715 \\
10.75 \%\end{array}$ & $\begin{array}{c}43.8504 \\
0.34 \%\end{array}$ & $\begin{array}{c}40.5605 \\
7.82 \%\end{array}$ & $\begin{array}{c}41.2153 \\
6.33 \%\end{array}$ & $\begin{array}{c}40.8768 \\
7.10 \%\end{array}$ \\
\hline 1998 & 33 & $\begin{array}{c}36.1841 \\
9.65 \%\end{array}$ & $\begin{array}{l}36.8848 \\
11.77 \%\end{array}$ & $\begin{array}{c}34.1202 \\
3.39 \%\end{array}$ & $\begin{array}{c}35.7297 \\
8.27 \%\end{array}$ & $\begin{array}{c}35.4152 \\
7.32 \%\end{array}$ & $\begin{array}{c}35.5751 \\
7.80 \%\end{array}$ \\
\hline
\end{tabular}

\section{Conclusions}

The combination prediction methods based on weighted coefficients determined by prediction result of single method is introduced in aviation accident prediction. In these methods, the weighted coefficients are mainly calculated by the arithmetic average method, error square sum reciprocal method and error standard deviation reciprocal method. The result of practical example shows that, on the basis of Gray method, simple regression method and ARIMA method, using the proposed combination method to predict the flight mishap of USAF can have a good prediction result with strong stability and anti-interference.

\section{References}


[1] B. N. Xu. Introduction to flight safety evaluation. Beijing: Blue Sky Press, (2005)

[2] H. L. Lu. Research of military aircraft accident. Beijing: National Defense Industry Press, (2003)

[3] J. M. Bates, C. W. J. Granger. The combination of forecasts. Operational Research Quarterly, 20(1), (1969), 451-468

[4] B. N. Xu. The initial forecast of the fatal accident rate per 10 thousand flight hour by means of the theory of grey system. Journal of Beijing Institute of Aeronautics and Astronautics, 14(2), (1988), 149-156

[5] G. X. Xu. Statistical forecasting and decision-making. Shanghai: Shanghai University of Finance and Economics Press, (2008) 